\title{
Una experiencia educativa desde la innovación docente universitaria a la intervención social penitenciaria
}

\section{An educational experience from University teaching innovation to the prison social intervention}

\author{
María del Carmen Sánchez Miranda \\ Universidad de Jaén. \\ mmiranda@ujaen.es
}

Recibido 11/12/2019 Revisado 02/01/2020

Aceptado 02/01/2020 Publicado 31/01/2020

\section{Resumen:}

El presente texto recoge la experiencia de innovación educativa a partir de una iniciativa de formación cooperativa y con aprendizaje basado en proyectos tutorizados, originada en el seno de la asignatura Antropología Urbana del Grado en Trabajo Social de la Universidad de Jaén, motivada por los intereses del alumnado y resultado de una profunda y madurada colaboración con un grupo de profesionales del Centro Penitenciario de Jaén.

A raíz de uno de los ejes temáticos de la asignatura, los estudiantes demandaron una experiencia formativa teórico-práctica paralela y complementaria a sus estudios, que integrara elementos de la realidad cotidiana del ámbito penitenciario: un espacio en el que gran parte de los egresados de esta disciplina podrán desempeñar su labor como profesionales de la acción social.

El ejercicio del Trabajo Social se configura como una especialidad que necesariamente ha de vincular la teoría con los requerimientos prácticos para su intervención con colectivos específicos de atención, cuanto más, en un sector tan especializado como las prisiones, cuyas características establecen una propia y particular cultura.

Sugerencias para citar este artículo

Sánchez Miranda, María del Carmen (2020). Una experiencia educativa desde la innovación docente universitaria a la intervención social penitenciaria. Tercio Creciente, 17, págs. 21-31. https://dx.doi.org/10.17561/rtc.n17.2

SÁNCHEZ MIRANDA, MARÍA DEL CARMEN. Una experiencia educativa desde la innovación docente universitaria a la intervención social penitenciaria. Tercio Creciente, enero 2020. $\mathrm{n}^{\mathrm{o}} 17$, pp. 21-31. https://dx.doi.org/10.17561/rtc.n17.2 


\begin{abstract}
:
The present text collects the experience of educational innovation from an initiative of cooperative training and learning based on tutored projects, originated in the bosom of the subject Urban Anthropology of the degree in Social Work from the University Jaén, motivated by the interests of the students and the result of a deep and mature collaboration with a group of professionals coming from Jaén Penitentiary.
\end{abstract}

As a result of the study of one of the central themes of the subject, students demanded a parallel theoretical-practical training experience and complementary to their studies, incorporating elements of prison daily reality: an area in which large part of graduates on this discipline will be able to carry out their work as social action professionals.

The practice of Social Work is configured as a specialty that necessarily has to link theory with practical requirements for its intervention within specific attention groups, specially in a sector as specialised as prisons, whose characteristics establish a particular and own culture.

\title{
Palabras Clave/Key words
}

Innovación educacional, Prisión, Trabajo Social, Universidad / Educational innovation, Prison, Social Work, University

\section{Sugerencias para citar este artículo}

Sánchez Miranda, María del Carmen (2020). Una experiencia educativa desde la innovación docente universitaria a la intervención social penitenciaria. Tercio Creciente, 17, págs. 21-31. https://dx.doi.org/10.17561/rtc.n17.2

SÁNCHEZ MIRANDA, MARÍA DEL CARMEN. Una experiencia educativa desde la innovación docente universitaria a la intervención social penitenciaria. Tercio Creciente, enero 2020. no 17, pp. 21-31. https://dx.doi.org/10.17561/rtc.n17.2 


\section{Introducción}

La institución universitaria, en múltiples ocasiones, adolece de reciprocidad y coordinación con los recursos y agentes sociales donde el futuro graduado desarrollará la profesión en la que se ha formado en la academia, detectando durante su periplo educativo la necesidad de una mayor interrelación, conocimiento e inmersión en las particularidades de cada sector de intervención en la sociedad.

Junto a esta casuística, las cárceles se caracterizan por un difícil acceso y una especial mitificación de la labor realizada intramuros, por lo que nos encontramos ante dos instituciones cuyas puertas de entrada y salida parten de la necesidad de un contraflujo de intercambio de saberes y de apertura de experiencias, donde "toda esta filosofía no se queda sólo en la institución penitenciaria; "implica" a la sociedad en ese proceso, "abre" la prisión a la comunidad. No se puede educar para la sociedad al margen de la sociedad" (Beltrán, 2010: 6).

En esta brecha surgió la idoneidad de llevar a cabo el proyecto de innovación docente ${ }^{1}$ que aquí mostramos, basado en la oportunidad de tender un puente desde la formación en las aulas hasta la intervención profesional del alumnado en Instituciones de Bienestar Social, a través la implementación de lo aprendido y mediante la elaboración de proyectos tutelados: una de las salidas profesionales de los futuros Graduados en Trabajo Social y línea de trabajo fundamental durante el bienio de este proyecto de innovación educativa.

Bajo el principio de corresponsabilidad institucional, el equipo de profesionales participantes en el proyecto, tanto por parte de la Universidad de Jaén, como por parte del Centro Penitenciario de Jaén, han arbitrado los mecanismos que permitan asegurar la adecuada formación y tutorización del aprendizaje de los estudiantes en el contexto de la intervención profesional con población reclusa, con el fin primordial de la reeducación y reinserción penitenciaria. Procurando una formación que trate de:

(Re)formar al individuo para asegurar el contacto con la comunidad que le recibirá cuando finalice su condena y proyectar en ésta los comportamientos que debe mantener para disfrutar de su vida en el exterior, evitando los posibles efectos perniciosos que le hayan provocado su estancia en prisión (Sánchez y Serrano, 2018: $63)$.

\section{Desarrollo}

\subsection{Antecedentes:}

El proyecto de innovación docente donde nos situamos se denominó "Cultura carcelaria e intervención social: un contexto de participación e innovación para el

\footnotetext{
${ }^{1}$ Basado en el proyecto de innovación docente denominado "Cultura carcelaria e intervención social: un contexto de participación e innovación para el aprendizaje en la Universidad".
} 
aprendizaje en la Universidad", actividad que ha procurado como fuentes inspiradoras que "el estudiante se convierte en consumidor y el conocimiento universitario es predominantemente profesionalizante que deviene de las necesidades del modo de producción, y se mantiene relativamente descontextualizado de las necesidades del tejido social y de la autorrealización" (Ramírez, 2017: 2) y partiendo desde el "análisis de la disciplina, Trabajo Social, abocada al tratamiento de la persona privada de libertad, infiriendo posicionamientos y postulando una disposición al abordaje que procura un encuentro con la dignidad de la persona" (Acevedo, 2003: 15).

Esta iniciativa estuvo dirigida hacia el Grado en Trabajo Social y se implicaron tres Departamentos -a su vez, en tres Áreas de Conocimiento que imparten docencia en la titulación-:

- Departamento de Antropología, Geografía e Historia (Área de Antropología Social).

- Departamento de Psicología (Área de Trabajo Social y Servicios Sociales).

- Departamento de Organización de Empresas, Marketing y Sociología (Área de Sociología).

Por su parte, el proyecto afectó como población diana a ciento veintitrés estudiantes matriculados en la titulación y a siete asignaturas, pertenecientes a los siguientes:

- $1^{\circ}$ curso: Antropología Social y Cultural (básica).

- $2^{\circ}$ curso: Antropología Urbana (obligatoria) y Fundamentos de los Servicios Sociales (obligatoria) / Trabajo Social y Mediación en Situaciones de Conflicto (obligatoria).

- $3^{\mathrm{er}}$ curso: Trabajo Social, Animación Sociocultural y Participación (obligatoria), Estructura de los Servicios Sociales (obligatoria) e Interacción Social a través del Trabajo Social de Grupos (obligatoria).

Un mosaico de profesionales, materias, áreas e instituciones implicadas que han promovido la acción formativa reflexiva y la dimensión crítica de la intervención desde las políticas sociales "poniendo el foco en las contradicciones entre una legislación penitenciaria que fomenta la educación y una lógica de funcionamiento que la dificulta" (Osuna, 2019: 279).

\subsection{Objetivos:}

Con una finalidad general basada en "la necesidad de comprender los códigos y normas que rigen la vida penitenciaria y que están recogidos en un complejo corpus legislativo" (Osuna, 2019: 279) para poder cuestionar el espacio estudiado, con una mirada educativa nueva, y precisando que "el conjunto de dimensiones a tener en cuenta para que la necesidad de innovar no se convierta en una 'moda', sino en una transformación paulatina, sostenible y con sentido de la práctica educativa y las finalidades de la educación" (Sancho, 2018: 14). Esto es, facilitando el hecho de: 
La educación liberadora es problematizadora, concientizadora y de hecho humanista y humanizadora. En esencia, esta perspectiva busca la profundización de la toma de conciencia del hombre sobre su realidad, bajo la exaltación de su condición de sujeto activo de su propia vida y existencia (Areiza, 2018: 19)

Desde los fundamentos de las premisas teóricas iniciales, el proyecto se materializó en una serie de objetivos generales y específicos, a conseguir durante el bienio de su puesta en marcha:

a) Objetivos generales:

- Preparar al alumnado del Grado en Trabajo Social en actitudes y aptitudes básicas para la intervención en centros de internamiento cerrados.

- Implementar la metodología del aprendizaje universitario a la praxis e idiosincrasia del medio penitenciario.

- Mejorar la formación especializada del perfil profesional del Graduado en Trabajo Social hacia las demandas actuales del mercado laboral.

b) Objetivos específicos:

- Sensibilizar a la población sobre la situación de vulnerabilidad y exclusión que origina la prisión y la necesidad de creer en la reeducación y reinserción.

- Dar a conocer la dinámica de funcionamiento del medio penitenciario.

- Analizar las principales consecuencias de la vida en prisión: la prisionización del recluso.

- Mostrar las posibilidades de los programas de tratamiento penitenciario.

- Desmitificar la cárcel como espacio inaccesible y hostil para la acción social.

- Dotar al alumnado de conocimientos, herramientas e instrumentos para el ejercicio profesional de la reeducación penitenciaria.

\subsection{Contenidos:}

Durante el bienio comprendido en este proyecto se ha procurado un abordaje integral hacia la cultura carcelaria y a la redefinición del tratamiento en las prisiones y la metodología para elaborar y diseñar acciones de intervención en el medio penitenciario, mediante aprendizaje basado en proyectos y con un proceso formativo cooperativo traducido en "una estructura de interacción diseñada para facilitar la consecución de un determinado objetivo, a través de la cual las personas trabajan en grupo, y donde el docente controla completamente la situación" (Pérez, 2013: 10). Así pues: 


\section{se invita a la participación, y las evidencias y producciones del alumnado se reparten por el centro incluso una vez finalizado el proyecto (Moreno, Tirado, López-Peláez y Martínez, 2017: 131).}

Los contenidos específicos abordados han estado compuestos por los siguientes espacios temáticos:

- Bloque $n^{\circ}$ 1: Introducción y aportes metodológicos para el diseño de proyectos en centros penitenciarios.

- Bloque $n^{\circ}$ 2: El cumplimiento de las penas desde el punto de vista del Derecho Penitenciario.

- Bloque $n^{\circ} 3$ : Los programas de tratamiento penitenciario y la preparación para la vida en libertad.

- Bloque $n^{\circ} 4$ : Vida cotidiana en un centro penitenciario: el caso del C.P. de Jaén.

- Bloque ${ }^{\circ} 5$ : Programa Unidad Terapéutica y Educativa.

- Bloque $n^{\circ}$ 6: Hacia la redefinición de un nuevo modelo: Programa Módulos de Respeto.

- Bloque $n^{\circ}$ 7: Módulos de Respeto: organización y funcionamiento. Delincuencia femenina: Módulos de Mujeres.

- Bloque n 8: Intervención social con población penitenciaria.

- Bloque $n^{\circ}$ 9: La cárcel como elemento de desarticulación de la socialización. Una propuesta desde la intervención de los agentes externos.

- Sesión práctica: Taller de debate con internos del C.P. de Jaén.

- Bloque $n^{\circ} 10:$ Proyecto de intervención en el medio penitenciario.

- Bloque $n^{\circ} 11$ : Seguimiento y evaluación de los trabajos de innovación tutelados.

Este compendio de temas de trabajo ha mantenido una formación transversal en valores formativos tales como "la motivación, el esfuerzo, la voluntad, etc., serán palabras claves con el propósito de fomentar la autorrealización, la crítica constructiva personal y la consecución de los logros establecidos" (Moreno, 2011: 15).

\subsection{Metodología y temporalización:}

En el contexto de la corresponsabilidad institucional indicada con anterioridad y la cooperación entre profesionales para llevar a buen término los objetivos planteados, nos basamos en un modelo en el que "el profesor mantiene el control de su clase. Para ello, el docente puede utilizar estrategias específicas, que ayuden a facilitar las interacciones grupales. El objetivo puede ser un producto específico" (Pérez, 2013: 11), resultado 
especifico que se materializa en la planificación, diseño y tutelaje de proyectos de intervención socioeducativa adaptados a las peculiaridades de la población reclusa. La marca y clave de nuestro quehacer ha sido abanderar "los derechos humanos, el prisma con el que ver conflictos y problemas, el baremo con que medir bondad y justicia de las situaciones que crean, provocan y mantienen los seres humanos" (Sánchez y Caño, 2012: 179).

Aplicando el método a la calendarización planteada, se comenzaron durante los dos primeros meses del proyecto las tareas de difusión y captación de participantes, con el fin de disponer de solicitudes suficientes para cubrir las sesenta plazas que había previsto ofertar -y poder contar con una lista de potenciales reservas-. Desde el equipo docente de la Facultad de Trabajo Social implicado, se dinamizó la iniciativa a través de las diferentes asignaturas afectadas, ofreciendo a los estudiantes información sobre el procedimiento a seguir para su curso con máximo aprovechamiento. Ante la gran demanda de solicitudes, se optó por no desestimar ninguna y buscar un espacio docente donde atender la motivación del alumnado, cuyo número de matrículas ascendió a ciento veintitrés, más del doble de lo planificado.

A continuación, se realizó la puesta en marcha, estructurada en dos fases, correspondientes a los dos años del bienio:

- $1^{\text {er }}$ año: Implementación de un programa de formación especializado (bloques temáticos $\left.n^{\circ} 1 a^{\circ} 9\right)$, paralelo a las enseñanzas de Grado en Trabajo Social.

- $2^{\circ}$ año: Se retomó el trabajo iniciado en el primer año del bienio y puntualizado en el marco de contenidos de los dos últimos bloques temáticos ( $n^{\circ} 10$ y $\left.n^{\circ} 11\right)$, para trabajar por proyectos y bajo tutorías individualizadas.

Junto a las sesiones presenciales cada semana (comportando una sesión semanal de tres horas de duración), el alumnado matriculado también trabajó mediante un espacio online en la plataforma de Docencia Virtual ILIAS de la Universidad de Jaén (PID201113_CULTURA CARCELARIA E INTERVENCIÓN SOCIAL), herramienta docente de apoyo durante todo el desarrollo del proyecto.

En dicha plataforma los estudiantes dispusieron de acceso a los materiales de cada bloque temático y, durante la segunda fase, pudieron cursar la entrega del proyecto de intervención tutelado y contar con espacios de reflexión conjuntos fuera de aula.

Debido al numeroso grupo de personas inscritas, un aspecto fundamental en la presente iniciativa ha sido el fomento de la responsabilidad a asumir por parte de los participantes, los cuales han tenido que comprometerse al aprovechamiento de las dos fases del proyecto (programa de formación y diseño de proyecto de intervención complementado con el espacio virtual del mismo en la plataforma ILIAS de Docencia Virtual-), con una fuerte presencia del trabajo tutelado, ya que:

las ventajas que ofrece la utilización de las tutorías de iguales como estrategia docente están centradas en que aumenta su motivación con respecto al aprendizaje, facilitan la 
adquisición de competencias tecnológicas y metodológicas y favorecen el desarrollo de estrategias de aprendizaje (Pino y Soto, 2010: 162).

El método de implementación la iniciativa se ha estructurado fundamentalmente en las siguientes etapas: período de aprendizaje dirigido -y cooperativo-, trabajo autónomo del alumnado y seguimiento tutelado del aprendizaje basado en proyectos, las cuales se suceden desde el inicio de primer año del proyecto de innovación, procurando:

un enfoque educativo que fomenta que los estudiantes "aprendan a aprender" y que trabajen de manera colaborativa en grupo para buscar soluciones a un problema real; el APB debe ser una de las herramientas fundamentales para el desarrollo de las competencias (Ausín, Abella, Delgado y Hortigüela, 2016: 32).

Todas las personas implicadas en este proyecto (ciento veintitrés alumnos iniciales, siete profesores de la Universidad de Jaén y diez profesionales de Instituciones Penitenciarias) han partido de la premisa de un método de trabajo reflexivo que permitiera:

estar alerta, hay que entender los fenómenos que nos rodean, hay que analizar lo que tenemos que preservar, lo que tenemos que erradicar, lo que tenemos que transformar, lo que tenemos que mejorar, lo que tenemos que soñar y proyectar, y todo lo que ello conlleva (Sancho, 2018: 15).

\subsection{Resultados y evaluación del proceso:}

El resultado prioritario de la puesta en marcha del proyecto de innovación docente no fue otro que ir más allá de las aulas, traspasar lo escrito en los libros y entrenar el aspecto emocional y personal del respeto y la dignidad de las personas privadas de libertad, que habitan en un contexto hostil y alejado de toda realidad sociocultural cercana y conocida, tratando:

compromiso en la mirada, sobre una cultura, sobre la particularidad devenida en generalidad, por la acción o la omisión de políticas específicas, dará un encuadre al cual el profesional se aproxima con responsabilidad, integrando acciones, con apertura de criterios, con un rol protagónico y crítico de la responsabilidad a la que se enfrenta (Acevedo, 2003: 26-27).

Cuantitativamente, se han destacado resultados que han impactado en dos áreas principales:

- Referente a la propia formación del alumnado y a la creación de material docente: un volumen de setecientas ochenta y seis páginas.

- En cuanto a la proyección externa y reciprocidad social obtenida: una vez estudiada la viabilidad y el interés de los participantes para la puesta en marcha real de acciones en el medio penitenciario, por parte del alumnado de la Universidad de Jaén en el Centro Penitenciario de Jaén, se han tutorizado en profundidad cinco de estas propuestas y se 
han llevado a cabo en prisión, a través de la colaboración de una organización no gubernamental $^{2}$.

Respecto a la evaluación se ha procurado una "evaluación continua y formativa es avalada por una revisión del proceso de aprendizaje del alumnado, lo que permite que haya una mejora del mismo, siendo un aspecto central de nuestra acción formativa" (Santos, Castejón y Martínez, 2012: 75), así como una "evaluación formativa entendemos aquella que está orientada al aprendizaje del alumnado, como proceso" (Santos et al., 2012: 77).

Los indicadores que se han tenido en cuenta en la evaluación han sido los siguientes:

- Número de solicitudes de alumnos interesados en participar en el proyecto de innovación docente.

- Nivel de participación del alumnado seleccionado en las actividades programadas en el calendario de ejecución.

- Grado de implicación por el alumnado y el profesorado participante durante las diferentes fases de implementación.

- Nivel de cumplimiento de los objetivos planteados.

- Grado de satisfacción del profesorado participante y de los colaboradores externos.

- Número de proyectos reales implementados en el centro penitenciario.

Aunque dentro de los resultados de la evaluación se extrae el dato negativo del abandono de un $40 \%$ de los alumnos inscritos al inicio de la actividad (habiendo quedado prácticamente el número de estudiantes con los que estaba previsto trabajar en un principio), esta cifra resulta de positiva lectura, al no haber desestimado ninguna de las solicitudes registradas y quedando un grupo con verdadero compromiso de trabajo y que, fehacientemente, ha mostrado sumo interés en la superación de los ítems de evaluación de previstos para el alumnado:

- Nivel obtenido por el alumnado durante la elaboración del trabajo tutelado.

- Calidad de los resultados plasmados en el material docente compilado a la finalización del proyecto.

En consecuencia, el pilar fundamental de la ejecución exitosa del proyecto se ha correspondido con la pormenorizada coordinación del grupo y el cumplimiento de la planificación y calendario establecidos. Todos los trabajos elaborados por los estudiantes

\footnotetext{
${ }^{2}$ A través de Cáritas Jaén en su proyecto de reclusos denominado "Nazaret"; iniciativa que, desde la implementación del proyecto de innovación docente a la actualidad, da cabida a alumnado de la Universidad de Jaén para realizar actividades formativas tuteladas de voluntariado en el Centro Penitenciario de Jaén.
} 
han sido tutelados por el equipo de miembros de la Universidad de Jaén, procurando una atención personalizada en la fase de elaboración y redacción del proyecto de intervención en el medio penitenciario.

\title{
3. Conclusiones
}

Aún considerándose una acción educativa concreta y contextualizada en el seno de una universidad, esta iniciativa de innovación docente ha coadyuvado en la fractura institucional del conocimiento, atención y comprensión de las periferias sociales donde están ubicados los establecimientos penitenciarios y sus habitantes, con el acercamiento a:

\begin{abstract}
las personas, en función de diferentes aspectos como son el género, el contexto, las expectativas de libertad y el tiempo de condena, desarrollan tácticas discursivas y prácticas diversas: de adhesión al modelo normativo, de identificación instrumental y de resistencias contestatarias y contrahegemónicas (Moreno, 2017: 534).
\end{abstract}

Por tanto, se ha tratado de generar una experiencia de innovación educativa que ha preparado a los estudiantes para el trabajo como profesionales de la acción penitenciaria, entrenándoles en mecanismos de apoyo a la erradicación de la delincuencia y la capacitación en herramientas que faciliten la incorporación a la sociedad, desarrollando acciones formativas integrales que favorezcan la inserción sociolaboral, familiar y comunitaria de los internos en establecimientos de cumplimiento penitenciario y articulando una constante retroalimentación con la realidad a lo largo del desarrollo de la presente iniciativa, fomentando el espíritu y la sensibilidad de "frente a los encarcelados no nos atañe averiguar ni juzgar, sino ayudar" (Sánchez y Caño, 2012: 205).

\section{Referencias}

Acevedo, J. A. (2003). Reflexiones acerca del Trabajo Social en las cárceles. Buenos Aires, Argentina: Espacio Editorial.

Areiza, E. (2018). Educación de calidad desde la perspectiva de los derechos humanos. Sophia, 14 (2): 15-23. DOI: https://doi.org/10.18634/sophiaj.14v.2i.778

Ausín, V., Abella, V. Delgado, V. y Hortigüela, D. (2016). Aprendizaje basado en proyectos a través de las TIC. Una experiencia de innovación docente desde las aulas $\begin{array}{lllll}\text { universitarias. } & \text { Formación } & \text { Universitaria, } & 9 & \text { (3): }\end{array}$ DOI: https://doi.org/10.4067/S0718-50062016000300005

Beltrán, J. (2010). La educación en prisiones. Elemento fundamental del tratamiento penitenciario. Educar(nos), 52: 5-8. 
Moreno, M. I., Tirado, A., López-Peláez, M. P. y Martínez, M. (2017). Investigación basada en las artes como investigación educativa: análisis de una experiencia en el Colegio San Isidro en Guadalén. Educatio Siglo XXI, 35 (1): 125-144. DOI: https://doi.org/10.6018/j/286251

Moreno, F. M. (2011). Proyecto de innovación educativa sobre la formación para las personas que acceden a la prueba de acceso a la universidad para mayores de 25 años. Revista de Comunicación Vivat Academia, 116: 118. https://doi.org/10.15178/va.2011.116.1-18

Moreno, G. (2017). Subjetividades y tácticas entre rejas: una etnografía del consumo de drogas y los dispositivos de tratamiento en prisión. En Vicente, García, P. y Vizcaíno, A. (coords.), Antropologías en transformación: sentidos, compromisos y utopías (pp. 524-536). Valencia, España: Universitat de Valencia.

Osuna, C. (2019). "Yo allí soy feliz, voy de lunes a viernes sin faltar": Reflexiones desde una etnografía escolar en una cárcel de mujeres. AIBR, Revista de Antropología Iberoamericana, 14 (02): 277-298. DOI: https://doi.org/10.11156/aibr.140206

Pérez, S. (2013). El teatro musical como vehículo de aprendizaje: un proyecto de innovación docente en la universidad. DOI: https://doi.org/10.6035/Sapientia77

Pino, M., Soto, J. (2010). Ventajas e inconvenientes de la tutoría grupal como estrategia docente. Bordón, 62 (1): 155-166.

Ramírez, J. V. (2017). ¿La universidad para profesionalizar o para el desarrollo cultural? Sophia, 13 (1): 1-3. DOI: https://doi.org/10.18634/sophiaj.13v.1i.696

Sánchez, C, Caño, X. (2012). Voluntarios en prisión. Ciudadanía en la sombra. Madrid, España: PPC.

Sánchez, M. C., Serrano, S. (2018). Modelando barrotes. Un análisis sobre el taller de alfarería en la Unidad Terapéutica y Educativa del Centro Penitenciario de Jaén. En Cueva, M. L. y Vico A. F. (Eds.), Prácticas artísticas multidisciplinares y alternativas (pp. 61-82). Jaén, España: AASA.

Sancho, J. M. (2018). Innovación y enseñanza. De la "moda" de innovar a la transformación de la práctica docente. Educação, 41 (1): 12-20. DOI: https://doi.org/10.15448/1981-2582.2018.1.29523

Santos, M. L., Castejón, F. J. y Martínez, L. F. (2012). La innovación docente en evaluación formativa y metodología participativa: Un proyecto compartido a raíz de la implantación de los nuevos grados. Psychology, Society, \& Education, 4 (1): 73 86. https://doi.org/10.25115/psye.v4i1.482 
Revista de Estudios en Sociedad,

Artes y Gestión Cultural

Número 17

Enero 2020

Investigación
DOI: https://dx.doi.org/10.17561/rtc.n17.2 